\title{
«Que en estos nuestros reinos Ilamábamos...»: a la búsqueda de una naturalización coeva de títulos y fórmulas de tratamiento en la traducción de la dramaturgia estuarda \\ "That we in these our kingdoms did call...": In search of a coeval naturalization of titles and terms of address in translating Stuart plays
}

\author{
Juan José Calvo García de Leonardo \\ calvojj@uv.es \\ Universidad de Valencia
}

Fecha de recepción: 29 de octubre de 2018

Fecha de aceptación: 26 de septiembre de 2019

Resumen: La traducción de textos no-contemporáneos, 'diacrónicos', se puede plantear desde la lealtad romántica al original hasta la absoluta domesticación. Centrándonos en la onomástica de los títulos heredados y adquiridos y en las fórmulas de tratamiento, proponemos una naturalización histórica y cultural, de modo que la reacción del público ante la puesta en escena de la traducción sea lo más equivalente posible a la del público de la cultura de partida, hoy en día.

La parte teórica arranca con la presentación y explicación de lo que entendemos como tres modos básicos de traslación, junto con la argumentación de nuestra propuesta metodológica. A continuación, se expone una taxonomía de títulos heredados o adquiridos y de las fórmulas de tratamiento posibles en los textos originales y su tratamiento traductológico, según nuestras premisas.

La parte práctica se inicia con una breve comparación de las versiones que cuatro traducciones canónicas, al alemán, el francés, el italiano y el español, dieron a dos títulos heredados o adquiridos en dos comedias, dos obras históricas y una 'problem comedy' shakesperianas. Pero nuestro corpus propiamente hablando consta de seis títulos heredados o adquiridos (Lord, Lady, Sir, Gentleman, Mr., Mrs.), veinte fórmulas de tratamiento de respeto, entre pares, incluyendo seis insultantes o degradantes (Your Grace, Lady, Your Ladyship, Your Worship, Worshipful, Right Worshipful, Right honourable, Sir, Fellow, Goodman/Goodwife, Master, Mistress, Madam, Miss, Rascal, Rogue, Sirrah, Wench, Hussy, Slut) y cuatro fórmulas paródicas o burlescas (Your Mastership, Your Solicitorship, His Wise Head, Reverend Ladies), extraídos de tres comedias 'estuardas' (Carlos I y Carlos II) del siglo 
XVII: A new way to pay old debts (1633) de Philip Massinger, The country wife (1675) de William Wycherley y Love for love (1695) de William Congreve.

Palabras clave: onomástica, equivalencia histórica y cultural, traducción, naturalización, comedia del inglés moderno temprano.

Abstract: The translation of non-contemporary, 'diachronic' texts can be undertaken from the tenets of Romantic loyalty to absolute domestication. Focusing upon the onomastics of inherited and acquired titles and upon the forms of address, we propose a historical and cultural naturalization, in such a way that the reaction of the audience of the translated version be as equivalent to that of the present day source culture audience as possible.

The theoretical part starts off with the presentation and explanation of what we understand to be the basic three translation procedures and the discussion of our methodological proposal. Then we expound a taxonomy of inherited or acquired titles and of address forms that may be found in the original texts alongside with their translational approaches, according to our premises.

The practical part begins with a brief comparison of the versions of four 'canonical' translations into German, French, Italian and Spanish of two inherited or acquired titles in two Shakespearian comedies, two history plays and one problem comedy. This being said, our actual corpus consists of six inherited or acquired titles (Lord, Lady, Sir, Gentleman, Mr., Mrs.), twenty forms of address showing respect, among peers, and including six abusive or degrading terms (Your Grace, Lady, Your Ladyship, Your Worship, Worshipful, Right Worshipful, Right honourable, Sir, Fellow, Goodman/Goodwife, Master, Mistress, Madam, Miss, Rascal, Rogue, Sirrah, Wench, Hussy, Slut) and four parodic or farcical forms of address (Your mastership, Your Solicitorship, His Wise Head, Reverend Ladies), taken from three Stuart (Charles I, Charles II) comedies of the 17th century: A new way to pay old debts (1633) by Philip Massinger, The country wife (1675) by William Wycherley y Love for love (1695) by William Congreve.

Keywords: Onomastics, Cultural and Historical Equivalence, Translation, Naturalization, Early Modern English Comedy 


\section{INTRODUCCIÓN}

Es un antiguo y aceptado error de traducción el aprovechar el 'falso amigo', el isomorfismo del inglés gracious y el español gracioso/a, para burlarse, en castellano, de Su Graciosa Majestad británica ${ }^{1}$. Como sabe cualquiera medianamente entendido en historia del derecho, gracious refiere a la prerrogativa aristocrática y/o real de gracia, que está por encima de la justicia y que es privativa de la voluntad de quien lo ejerce. En la cultura inglesa, el tratamiento de Your Grace corresponde a los duques que no sean hermanos del monarca y a los arzobispos ${ }^{2}$. Desde el punto de vista de la traducción, salvando el isomorfismo, translúcido cuando no opaco, de $S u$ Gracia, la única solución viable es la de la naturalización mediante substitución cultural. Y ahí se nos ofrece una opción múltiple: Vuecencia si se le habla a un duque no perteneciente a la familia real, Ilustrísima si es a un obispo o la híbrida Vuestra Excelencia reverendísima si se trata de un arzobispo... pero, en la Edad Moderna, quizás la opción más habitual fuera Vuecencia o, simplemente, Señoría ${ }^{3}$.

\section{MODOS DE TRANSLACIÓN}

Antes de proceder al análisis propiamente dicho, repasemos de manera muy breve las opciones básicas, mínimas, que se le presentan al traductor a la hora de acometer su labor.

\subsection{Modos básicos de translación}

En lo que se refiere a los títulos y a las fórmulas de tratamiento, de los tres vectores básicos de translación ${ }^{4}$, a saber: la 'apropiación', la traducción semántica por 'triangulación referencial' y la 'substitución', lo habitual viene siendo (desde la Edad Moderna en buena parte de Europa y desde la Edad Contemporánea en España) proceder a la apropiación -lo que Vinay y

${ }^{1}$ El mismo Astrana comete ese error en La primera parte del rey Enrique VI (en sus siglas 1H6) I, i, 103 al traducir my gracious lords como "mis graciosos señores".

${ }^{2}$ Desde el punto de vista histórico también era el tratamiento debido a los monarcas de Inglaterra hasta Enrique VIII (muerto en 1547) y a los de Escocia hasta el Acta de Unión que, junto con el Decreto de Unión y otras disposiciones legales, unió legalmente ambos reinos en 1707. Pero dichas particularidades o anteceden nuestro período de referencia (el siglo XVII) o lo sobrepasan geográficamente, puesto que sólo tratamos la dramaturgia dentro del reino de Inglaterra. En consecuencia, no hemos tenido que añadir (Gran) Señor/Señora o Alteza a las posibilidades de traducción contempladas en el título.

3 «Á los Rreies dezimos.Magestad: á los prinçipes Alteza; á los grandes Eszelenzia; á otros titulados, i obispos Señoria; (...). Soliase usar sin orden de otras adulaziones i encarezimientos: Manifico, Illustre, llustrisimo; ia se quitó tal abuso por prematica rreal, i se rreservó para los señores, i prelados.» Gonzalo Correa (1954, pg. 213).

${ }^{4}$ Utilizamos el término translación (que tomamos de la Translation de la Escuela de Leipzig) para evitar la redundancia de traducción como hiperónimo e hipónimo a la vez. 
Darbelnet (1958) distinguieran como emprunt y calque - ya sea in toto como 'adopción' (esperando que el lector sepa leer el nombre como en el idioma original, lo que en inglés ha sido históricamente improbable) ya sea parcialmente, como 'adopción' ortográfica y' adaptación' fonética u ortográfico-fonética. Hay una subvariante -llamada, en inglés, loan translation y que no pasa de ser una adaptación semántica- donde se traduce, elemento por elemento, el lema original, típicamente en compuestos transparentes, y donde el préstamo puede fosilizarse en 'xenismo cultural', como en los ejemplos alemanes Pfalzgraf "conde palatino", Kurfürst «príncipe elector» o Bürgermeister «burgomaestre».

Por cierto, que también hay otra variante, intermedia, aunque aquí no interesa directamente y que propongo catalogar como 'vectigal o perasíctona' $5 \mathrm{y}$, con mayor exactitud en este tipo concreto de referencia, como 'vectigocultural/emporiopedeusónima' 6 . En ella, se adopta una fórmula de tratamiento que, aunque extranjera, no es la de la cultura original, sino de una cultura interpuesta; por lo general será el francés desde el siglo XVIII hasta mediados del XX y el inglés a partir de entonces. Por dar un sólo ejemplo, en las versiones al castellano, escritas o audiovisuales, de originales que refieren a la cultura japonesa tradicional, aunque no se da con el préstamo samurái, es habitual encontrarnos con un Lord/Lady antepuestos al nombre del clan correspondiente, en vez de adoptar/adaptar, mediante transliteración y vía adaptación fonética, las auténticas fórmulas de tratamiento niponas, vgr. daimio o josei.

Lo que llamo 'triangulación', es decir: la conmutación lingüística que ejecutamos en lo que vulgarmente se conoce como 'traducción', apoyándonos en un referente físico o metafísico compartido por ambas lenguas y/o culturas, uniendo y cerrando el triángulo, apetece evocar el esquema con el que Ullmann (1951) modificó el de Ogden y Richards (1923), que los estructuralistas presentaron como condición necesaria (y suficiente) de traducción y que, desde Koschmieder (das Gemeinte en 1953, 1955), Koller (1979: 114 s. Begriff, gemeinter Sachverhalt), pasando por Neubert (1968, pgs. 21-33 objektive Realität), Reiss (1976, pp. 11-14 Redegegestand), pero también los generativistas y los especialistas de la teoría de la comunicación, se ha ido rebautizando, en cumplimiento del anabaptismo endémico de las ciencias humanas en general y de la lingüística en particular.

\footnotetext{
${ }^{5}$ Neologismo derivado del latín uectigal (ex portu) «derechos de entrada en un puerto» y del

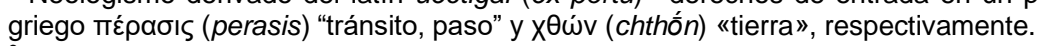

${ }^{6}$ Neologismo a partir del latín uectigal (ex portu) «derechos de entrada (en/de un puerto)» y

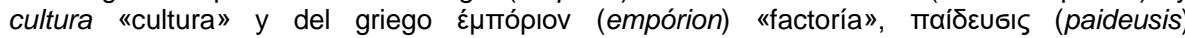

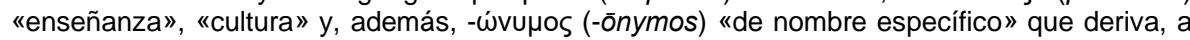
su vez, de övoua (ónoma) «nombre»,respectivamente.
} 
El tercer vector, el de la substitución en general y de la substitución cultural, corresponde a dos procedimientos/técnicas de Vinay y Darbelnet (1958). Lo que llamaron équivalence y que la escuela neerlandesa de Holmes prefirió calificar de permutation veinte años después; y lo que llamaron adaptation, que Levý defendía para "lo específico histórico y nacional" (1969, pgs. 92-102) y que Catford elevaría al rango de cultural untranslatability (1965, pg. 99). En el primero de los casos y con los nombres que queramos darle $^{7}$ el término se utiliza para lo que he llamado 'unidades de traducción global' (UTG), para los translemas de extensión extraordinaria, como los chistes, los insultos, las frases hechas, la paremia y demás conjuntos, superiores al rango de oración o no. Siempre supondrá un reemplazamiento puramente funcional, recurriendo de manera expresa al material lingüístico/cultural autóctono, ya sea sincrónico o diacrónico, lo que Vladova (1993, pp. 15 s., en Shuttleworth y Cowie, 2014, q.v.) Ilamó «intertemporal/cross-temporal translation», como es nuestro caso. En el segundo, siempre y cuando se trate de un elemento no transferible como la yukata japonesa que cita Catford (1965: 100), tenemos dos opciones: acudir a la 'apropiación' o intentar compensar ese void (Dagut 1978), esa laguna referencial, mediante la 'substitución' ... incluida esa «foca de Dios» inuit que famosamente/apócrifamente propusiera Nida para el Agnus Dei mediterráneo. Y en ello nos basamos aquí.

\subsection{Factores moduladores de la traducción historicista}

Dado que nuestra propuesta de traducción es, a la vez, historicista y filológica, la modulamos mediante dos factores correctores. Remedando las 'unidades aristotélicas' coetáneas de la dramaturgia durante la Restauración estuarda (que eran las prescriptivas por la influencia omnipresente de la cultura francesa), proponemos dos factores cohesivos, la coherencia de tiempo y la coherencia de espacio, como condicionantes previos de la translación en cada 'translema's.

La coherencia del 'tiempo' es la primera y la más importante. En la medida de lo posible, apetecemos que el término español escogido se documente en los textos $u$ obras lexicográficas antedatas 0 , al menos, coevas, para evitar la extemporaneidad anacrónica. A nuestros efectos, eso significaría que el término castellano que propusiéramos figurara en Nebrija, en Covarrubias e incluso, aunque sobrepasándose en algunos años, en 'Autoridades'; en su defecto, que figurara en cualquiera de los diccionarios bilingües que recoge el NTLLE hasta principios del siglo XVIII. Pero, como quiera que la consignación lexicográfica puede postponerse, incluso siglos, por las razones más peregrinas, a su aparición, centramos nuestra atención

${ }^{7}$ Entre ellos y hasta cierto punto, la Adäquatheit (Reiss, 1971, pp. 11, 22, 61, 68).

${ }^{8}$ Para la inserción del término en la traductología española vid. Santoyo, 1986. 
en el Oxford English Dictionary por un lado y, por el otro, en los dos corpora en-línea de la lengua española: el Corpus del nuevo diccionario histórico del español (CNDHE) y el Corpus diacrónico del español (CORDE), con preferencia del primero sobre el segundo debido a las notables carencias de este último ${ }^{9}$.

El segundo condicionante, el del 'espacio', intenta evitar que el término escogido en la lengua término para el translema de la lengua de origen, 'choque frontalmente' con el entorno físico del original, resultando extraterritorial o exóctono ${ }^{10}$. Este segundo condicionante no representará un obstáculo notorio en el teatro europeo anterior al siglo XIX.

La objeción de mayor calibre que se le puede plantear a esta apetencia nuestra es bífida. Por un lado, está la hipótesis de que una versión en el lenguaje del Siglo de Oro podría no ser entendida por el público asistente, con lo que la pretensión básica de la traducción que es el de la comunicación para beneficio social o de terceros («putaui mihi suscipiendum laborem utilem studiosi, mihi quidem ipsi non necessarium», como recalcaría Cicerón) ${ }^{11}$ quedaría anulada. Ya lo planteaba Levý (1969, pp. 69 s.) al afirmar:

Ebensowenig könnte ein moderner Übersetzer einen romantischen Dichter in der Sprache von Novalis oder Brentano übersetzen, eher wird er Andeutungen eines romantischen Stils aus den Sprachmitteln schöpfen über die die heutige Poesie verfügt. ${ }^{12}$

Nuestra pretensión es conseguir la 'isapergasía'13: la similitud/igualdad de efecto en el espectador de nuestra versión con respecto a la del público de la cultura de origen ante el texto en la lengua 1 (TL1) o lengua de partida. $\mathrm{Y}$, en la medida en que el original pueda suponer una alienación lingüístico/cultural, y presente arcaísmos lingüísticos y/o culturales para el público de cultura anglosajona del siglo XXI, que esta alienación encuentre su reflejo equivalente en el público de cultura española de hoy en día.

La segunda objeción es que el público rechace una representación historicista, acostumbrado como esta al travestismo de contenidos para agradar a postcolonialistas, feministas y demás y al kitsch en las formas ... lo que es particularmente notorio en los escenógrafos operísticos y su obsesión por la época de Entreguerras y/o la Guerra Fría. Ante esto no podemos hacer nada. Son ya demasiadas décadas las que han aceptado sin rechistar la

\footnotetext{
${ }^{9}$ En consecuencia, solamente recurriremos a CORDE si CNDHE ofrece citas posteriores o ninguna.

${ }^{10}$ Derivado del griego $\dot{\varepsilon} \xi \omega-(e x \overline{-})$ «fuera» y $x \theta \omega ́ v$ (chthốn) «tierra».

11 Cicerón, De optimo genere oratorum $\mathrm{V}, 13$.

${ }_{12}$ Aunque la lírica es género distinto: hace miles de años que la poesía no se recita, naturalmente, con bardo, skop o aedo, punteado por arpa o lira, ante un público congregado; y aun aquella era poesía épica.

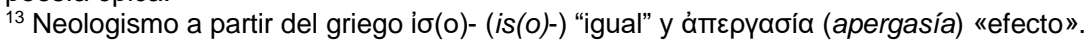


famosa antítesis de Schleiermacher de $1813^{14}$ de que hay que traer el autor al público y no el público al autor, de preferir la leche de bote a la leche materna, como si el público fuera, por naturaleza, párvulo.

Identificándonos con la escuela funcional en traducción y, de manera limitada, con la prevalencia del receptor final en el skopos de Vermeer y Reiss $(1996)^{15}$, lo que realmente perseguimos es una variante. De todas las definiciones de 'equivalencia', propuestas por traductólogos del siglo XX y que recogía Wilss (1977, pp. 157), nos quedamos con la de Jumpelt (Prinzip des äquivalenten Effekts "principio de efecto equivalente») y la de Koller (Wirkungsgleichheit «igualdad de efecto») ${ }^{16}$.

\section{TÍTULOS Y FÓRMULAS DE TRATAMIENTOS}

En primer lugar, se analizarán los títulos cognominales y agnominales, para luego centrarnos en el más voluminoso corpus de la pragmática de fórmulas de tratamiento.

\subsection{Relación de títulos y de fórmulas de tratamiento}

Los antropónimos que se plasman en los títulos y en las fórmulas de tratamiento, son cognominales y agnominales ${ }^{17}$, es decir:

a. Títulos hereditarios de la aristocracia o nombres cognominales $(<$ latín cognomen).

b. Títulos de apelación personal o nombres agnominales (< latín agnomen).

Estos últimos pueden subdividirse, según la consideración, en 'comunales' (vgr. los motes, alias y demás sobrenombres vulgares) ${ }^{18}$ y en

\footnotetext{
${ }^{14}$ En versión castellana de Valentín García Yebra: «O bien el traductor deja al autor lo más tranquilo posible y hace que el lector vaya a su encuentro, o bien deja los más tranquilo posible al lector y hace que vaya a su encuentro el escritor» (Vega, 1994, pp. 231). El original de Das problem des Übersetzens se puede leer, i.a., en Störig (1969, pp. 38-70).

${ }^{15}$ Cf. también Wilss (1977, pgs.168 ss.)

${ }^{16}$ Las citas que incluye Wilss son, por orden cronológico, de: Jakobson (1959), Jumpelt (1961), Güttinger (1963), Nida (1964), Tarnoczi (1967), Jäger (1968), Kade (1968), Levý (1969), Popovič (1971), Roganova (1971) y Koller (1972).

${ }_{17}$ Para esta clasificación, vid. i.a. Calvo 2013.

${ }^{18}$ A lo largo de toda la Edad Media y de manera particular en las sociedades germánicas (incluidos los escandinavos y los normandos) el mote o apelativo personal, a menudo con carácter burlesco, era moneda común. Destaca, por lo positivo, el apodo de Richard 'Coeur-delion'. («corazón de león»). Más habitual es el de su padre Henry Curtmantle («manto corto») o el de su hermano John Lackland («Sin tierra»). En años anteriores, lo vemos entre los anglosajones, como Ethelred 'the Unready' («el mal aconsejado») y entre los anglo-daneses, como Sveyn Forkbeard («barba hendida») o Harold Harefoot («pies de liebre»); en los años venideros, en Richard I 'Longshanks' («patas largas») o en el primero de los Tudor, Henry 'the Accountant' («el contable»), entre otros.
} 
'respiciales', de respeto ${ }^{19}$, que son los que aquí no interesan y que, según la alusión referencial, se subclasifican a su vez en:

a) Los títulos de respeto de la nobleza o 'agnominales respiciales patriciales' (como king, prince, duke, marquis/marchioness, earl, viscount, baron, baronet, knight)

b) Los títulos de respeto militares o 'agnominales respiciales graduales' (como admiral, vice-admiral, rear-admiral, commodore, commander, boatswain/bo'sun, ensign, midshipman etc.; general, colonel, major, captain, lieutenant, ensign, sergeant, corporal)

c) Los títulos de respeto de la santidad o 'agnominales respiciales sacrales' (como Saint, Blessed, Venerable, Servant of God)

d) Los títulos de respeto de la Iglesia o 'agnominales respiciales curiales' (como cardinal, archbishop, bishop, archpriest, archdean, dean, canon, abbot, abbess, prior, prioress y father, mother, brother o sister)

e) Los títulos de respeto del conocimiento o 'agnominales respiciales intelectuales' (como professor, doctor, master, bachelor, graduate)

f) Los títulos de respeto de la administración civil y/o de justicia o 'agnominales respiciales ministeriales' (como (Lord Chief) justice, judge, magistrate, judge of peace; governor, mayor, sheriff, bailiff, constable, etc.)

g) Los títulos/tratamientos de respeto de la sociedad burguesa o 'agnominales respiciales societales' (como Mr. (Mister), Mrs. (Mistress), Miss, Master, Goodman, Goodwife (Goody))

h) Los títulos/tratamientos de respeto de la familia o 'agnominales respiciales parentales' (como father, mother, uncle, aunt, grandfather, grandmother, dame, cousin/coz ${ }^{20}$, sin ser necesariamente familiares consanguíneos, ni tampoco afiliales, i.e. por matrimonio, sino, a menudo, afectivos y coloquiales.

2.2. Translación de los títulos y de las fórmulas de tratamiento

Las fórmulas de tratamiento entre ambas lenguas pueden:

a. Ser triangulables (con un referente físico o metafísico compartido) como FatherlPadre, Your Royal Highness/Su Alteza Real y otros.

b. Ser idioculturales/idiolingüísticos (como la intransferibilidad (salvo perífrasis, glosa o nota a pie de página) de infanzón o de hidalgo) ${ }^{21}$

\footnotetext{
${ }^{19}$ Del latín respicio «(yo) tengo en consideración».

${ }^{20} \mathrm{La}$ apócope familiar coz es propia de la Edad Moderna: nueve de las diez citas que registra el OED lo son de los siglos XVI-XVIII.

${ }^{21}$ Como ejemplo príncipe el siguiente. El lema hidalgo en El ingenioso hidalgo D. Quijote de la Mancha fue traducido como Knight-Errant por Thomas Shelton (publicado en 1612), como "Juncker" por Joachim Caesar (1648) y como «Edlen» por Ludwig Tieck (1799-1801); el lema se
} 
c. Ser plurirreferenciales (por ejemplo, Master es, a la vez: amo, señor y señorito, según las circunstancias)

d. Tener su 'reflejo' funcionalmente equiparable o no, en la otra cultura/lengua en contraste ya sea éste más o menos homónimo (como chief justice y justicia (mayor del rey), ya sea homólogo, como sheriff que, hasta la Edad Contemporánea, ni es comisario, ni sheriff, ni mucho menos chérif - como se atreve Astrana en KJpero sí alguacil (mayor): un término válido hasta en sus valores etimológicos como 'gobernador' en la España medieval musulmana con relación a lo que representaba el scir-zerefa (literalmente shire reeve "gobernador de condado») en el inglés antiguo

e. Ser isomorfos, e inducir, con ello, a error por inferencia semántica. Tal es el caso, -enormemente común en los rangos de la justicia y de la administración- de magistrate «A magistrate is an official who acts as a judge in law courts which deal with minor crimes or disputes» (Collins Cobuild English Dictionary) y magistrado «Funcionario que administra justicia en la Audiencia nacional, en los tribunales superiores de justicia, en las audiencias territoriales y provinciales o en el Tribunal Supremo» (DRAE) y que podríamos equiparar vgr. al de notario público y notary public que son absolutamente asimétricos en sus rangos sociales. Es lo que se daría, dentro de los dilemas traductológicos, cuando el elemento del 'mapa' de la L1 tiene una correspondencia formal pero no funcional con el elemento del 'mapa' de la L2: una correspondencia 'homológica' (Holmes 1978, pp. 75).

\section{CORPUS}

Damos comienzo al análisis `propiamente dicho del corpus con una mínima comparación antedata (apud Shakespeare) del tratamiento de títulos cognominales y agnominales a manos de reconocidos traductores antes de centrarnos en nuestro material.

\subsection{Los títulos y tratamientos en traducciones históricas shakesperianas}

Veamos las propuestas sobre dos títulos en la dramaturgia shakespeariana en cuatro traducciones 'canónicas', cronológicamente ordenadas: al alemán de Wilhelm Schlegel y del conde Wolf Heinrich von Baudissin (1789-1833), al francés de François-Victor Hugo (1859-1865), al

omite en la traducción francesa de César Oudin (1614, $1^{\text {a }}$ parte) y François Rosset $\left(1618,2^{\mathrm{a}}\right.$ parte) y se da como "cittadino» por Lorenzo Franciosini (1622, 1ㄹ parte; 1625, 2 $2^{\mathrm{a}}$ parte). Los diccionarios bilingües dan «noble» 0 «nobleman» 
italiano de Carlo Rusconi (1873-1874) ${ }^{22}$ y, como colofón, la española de Luis Astrana Marín (1932)23.

Los personajes son Mistress Kate Keepdown y Mistress Overdone de $\mathrm{MM}^{24}$ y Mistress Quickly (MWW, 1H4, 2H4); por el otro lado, Sir Andrew Aguecheek y Sir Toby Belch de TN. Las decisiones, son dispares, no solamente entre las cuatro lenguas y cinco traductores, sino incluso dentro de una misma lengua, obra y/o traductor. De los 20 casos, hay una clara mayoría de substituciones culturales (12) lo que representa un $20 \%$ por encima de las apropiaciones (8) y ninguna traducción semántica.

\section{Mistress}

Los dos traductores alemanes, Schlegel y el conde Von Baudissin, optan por la triangulación en ambos casos, pero con opciones distintas. Así el tratamiento de Mistress será «Frau» por razón de edad (que no de dignidad, que sería su valor etimológico, pues ambas son celestinas más o menos pregonadas) para Mistress Overdone de Measure for Measure y para la Mistress Quickly de ambas partes de Henry IV, mientras que Von Baudissin opta por «Jungfer» (es decir «doncella»), para referirse a la joven prostituta embarazada de Measure for Measure. Victor-Hugo triangula con el formal «Dame», excepto para Mistress Quickly donde apropia el anglicismo. Rusconi triangula siempre en "Madonna» y Astrana parece imitar la traducción francesa, al triangular "Señora» para los personajes de Measure for Measure y apropiar Mistress en Henry IV.

\section{Sir}

En el caso de este título agnominal masculino, Schlegel es sistemático y substituye culturalmente por «Junker», mientras que todos los demás prefieren la apropiación Sir.

\subsection{Nuestro corpus}

Nuestro corpus principal se centra (salvo A new way to pay old debts) en el último cuarto del siglo XVII, durante la Restauración de Carlos II

\footnotetext{
${ }^{22}$ No se han elegido las primeras traducciones. Por ejemplo, la así llamada Schlegel-Tieck Ausgabe es la 'clásica' para la cultura alemana, pero la de Christoph Martin Wieland (1762-1766) le precede en una generación. En el caso de las traducciones al español, se pueden consultar, entre otras obras de investigación, el primer capítulo de la tesis doctoral de Laura Campillo Arnaiz (2005). Salvo con Astrana Marín, se ha escogido el siglo XIX porque es el que asume el concepto romántico de la fidelidad al original y la subordinación del traductor al autor.

${ }^{23}$ La relación ha sido extractada y modificada de mi comunicación de 20 de octubre de 2016: «Voces con doblez, persona interpuesta: la bialienación en la traducción teatral diacrónica», presentada en el Congreso internacional Entresiglos. Literatura e Historia, Cultura y Sociedad, en homenaje al Prof. Dr. D. Joan Oleza, celebrado en Valencia del 19 al 21 de octubre de 2016.

${ }^{24}$ Utilizamos las siglas shakespearianas generalmente aceptadas.
} 
Estuardo, período que se halla encuadrado entre intransigencia de la República (1640-1660) y la agonía de las representaciones teatrales que resultan de la confluencia de la burguesía calvinista (véase el panfleto de Jeremy Collier Short View of the Immorality and Profaneness of the English Stage de 1698) y los gustos de la flamante casa de Orange. En orden cronológico, el corpus consta de las tres obras siguientes: $A$ new way to pay old debts (Philip Massinger, 1633), The country wife (William Wycherley, 1675) y Love for Love (William Congreve, 1695), todas ellas traducidas por nosotros, dentro del proyecto EMOTHE, siguiendo los mismos principios que aquí se exponen.

\section{A new way to pay old debts (1633)}

Lord Lovell, an English lord

Sir Giles Overreach, a cruel extortioner

Lady Allworth, a rich widow

The country wife (William Wycherley, 1675)

Mr. Horner, a gentleman

Mr. Harcourt, his acquaintance, enamored of Alithea

Mr. Dorilant, friend to Mr. Harcourt

Mr. Pinchwife, a newlywed gentleman

Mr. Sparkish, engaged to Alithea

Sir Jaspar Fidget, a noble gentleman

Mrs. Margery Pinchwife, a newlywed country lass

Lady Fidget, wife of Sir Jaspar

Mrs. Dainty Fidget, sister to Sir Jaspar

Mrs. Squeamish, granddaughter to Old Lady Squeamish and cousin to Lady Fidget

Old Lady Squeamish

Love for Love (William Congreve, 1695)

Sir Sampson Legend, Father to Valentine and Ben

Mrs. Foresight, second wife to Foresight

Mrs. Frail, sister to Mrs. Foresight, a woman of the town

Miss Prue, daughter to Foresight by a former wife, a silly, awkward country girl 
Además de los seis títulos de respeto, analizaremos catorce fórmulas de tratamiento, seis de vituperio y cuatro de imitación paródica que figuran en el corpus.

\subsubsection{Títulos de respeto. Análisis y propuesta de translación}

Los títulos hereditarios aristocráticos, cognominales, en nuestro corpus, son Lord y Lady (aunque el título femenino también puede ser adquirido por matrimonio y, por tanto, ser agnominal, como en el tipo siguiente). Los títulos personales, agnominales, son Sir (y Lady). Los títulos y tratamientos de respeto, en su caso, para la baja nobleza hidalga y para la burguesía, son: Sir, Gentleman, Mr. y Mrs.

Lord

Lord ya se había recibido en castellano, a finales del siglo XV, como préstamo ${ }^{25}$ exclusivamente referido a la cultura inglesa y se apropia.

25 CNDHE documenta el título lord (y también milor) en las Crónicas de los Reyes Católicos de Diego de Valera (1487-1488) [ed. Juan de Mata Carriazo, Madrid, Junta para la Ampliación de Estudios, 1927]:

«ovo certidumbre de milor Tamorlant lord Stanley, que es uno de los mayores señores de Inglaterra"

El título y la fórmula de tratamiento lady se documentan en español a partir de 1786, en la novela de Pedro Montegón, Eusebio [España] [Fernando García Lara, Madrid, Cátedra, 1998]:

«lady Lisle, tía suya, ... mandó arrestar a la misma lady y hacerle el proceso por haber dado asilo a un rebelde.»

El caso de Miss/miss es más complejo. CNDHE (y, parcialmente, CORDE) lo da:

a) como tratamiento (también en CORDE)

1786, Pedro Montegón, Eusebio [España] [Fernando García Lara, Madrid, Cátedra, 1998]:

"miss Rimbol, éste era el nombre del ama,»

b) en el sentido de "señorita", (también en CORDE)

1786, Pedro Montegón, Eusebio [España] [Fernando García Lara, Madrid, Cátedra, 1998]:

«ldo Henrique Myden, miss aderezó la mesa con limpios manteles y llamó a ella a su amo.»

c) en el sentido de «artista circense» (también en CORDE, no recogido en el NTLLE)

1884-1885, Clarín (Leopoldo Alas), La Regenta [España] [Gonzalo Sobejano, Madrid, Castalia, 1990]:

«a don Pompeyo le recordaban la cabellera rubia y estoposa de alguna miss de circo ecuestre;» (cita no recogida en CORDE)

d) en el sentido de «institutriz» (1897, en CORDE; no recogido en el NTLLE)

1891, Luis Coloma, Pequeñeces [España] [Rubén Benítez, Madrid, Cátedra, 1987]:

"¿Qué es esto, Miss? Hay que estudiar la lección de piano hasta las ocho».

e) en el sentido de "ganadora de concurso de belleza» (1941, en CORDE).

c1933, Ramón Magre, en la novela Un periodista [España] [Gonzalo Santonja, Madrid,

Ayuso, 1979]:

Hikma 18 (2) (2019), 37 - 65 


\section{Lady}

Ni CORDE ni CNDHE registran el anglicismo lady (como fórmula de tratamiento, vid. infra) antes de la novela Eusebio de Pedro Montegón en 1786. El NTLLE nos dará el 'Suplemento' de Domínguez de 1853. Al igual que haríamos con Lord, se apropia el título cognominal o agnominal Lady si bien lo culturalmente paralelo, en aquella época, habría sido "Doña".

\section{Sir}

Sir se puede substituir por "Don"26, que es el título castellano correspondiente, pero la apropiación Sir cumple con la coherencia temporal desde mediados del siglo XVI.

\section{Gentleman}

Se opta sistemáticamente por "hidalgo"27 como título cognominal/agnominal y como fórmula de tratamiento, no solamente por ser la obvia substitución cultural, sino porque caballero podía limitarse, en el español de entonces, a las órdenes militares o al armado caballero. Así se desprende desde su primera datación, c. 1140, en El Poema de Mio Cid. Hay que esperar al siglo XVIII, a Sobrino (1705) "Cauallero m. chevalier, gentilhomme de race noble» en su segunda acepción y a Stevens (1706), para hallar la correspondencia de cavallero con gentleman, aparte de la de cavalier y knight, trooper y horseman (NTLLE). Hidalgo como título se remonta a 1218 y el anónimo, Pesquisa de heredades en Tamayo y Santé, ordenada por Lope Díaz de Haro, García Lope y don Tello de Tamayo" (Colección diplomática de San Salvador de Oña): «Petro Petri de Tamayo qui erat cauallero / 7 et filiodalgo"; mientras que, como fórmula de tratamiento, vale desde 1379 - a1425 [s. XV (c1465)] Alfonso de Villasandino Poesías (Cancionero de Baena) [España] [Madrid, Visor, 1993]: «Señor Tello de Guzmán, /gentil fidalgo de onor, / dezid, amigo e señor.»

Mr.

\footnotetext{
«En vez de hacer una interviú vacía, intrascendente, ramplona, a la posible Miss Barcelona, haría otra a Miss Trabajo, más viva, más interesante y plena de emoción humana».

EI NTLLE remitirá los diccionarios manuales de la Academia de 1927 para el primer sentido, añadiendo el valor de tratamiento en el de 1950 y el significado de ganadora de concurso de belleza en el de 1984.

${ }^{26}$ Astrana ofrece "Sir" (MWW, KJ, R2, 1H4,), «Sire» por probable interferencia con el francés $(A Y L)$ y, como fórmula de tratamiento, «señor» (1H4). La apropiación figura desde la anónima Crónica del Rey Henrico Octavo de Ingalaterra, de 1549-1554: «allí se halló un caballero del Rey, que se llamaba Sir Arequenebet».

${ }^{27}$ Astrana ofrece «noble» (KL), «caballero» (MWW, Shrew, Cymb) e «hidalgo» (TGV, Shrew).
}

Hikma 18 (2) (2019), 37 - 65 
Mr. (Mister) se substituye, de manera históricamente adecuada, por «Maese», lema citado por vez primera en 1464-1485, en el anónimo Libro de Acuerdos del Concejo Madrileño: «e Ferrando de la Piedra e Diego de Monçón e Maese Pedro e Rui Ferrández e Gonzalo Sánchez e Diego Rodríguez e Ferrand García, escriuanos públicos»; si bien Raphael Bluteau propondrá el italianismo missser/micer como traducción del Mr. inglés en su diccionario de 1716-1721.

Mrs. «Doña».

Mrs. (Mistress), la correspondiente forma femenina, se substituye por

En sí, los títulos de corpus no son, salvo Lady, problemáticos. La mayoría de los títulos del Renacimiento y de la Edad Moderna tienen equivalentes directos en el resto de Europa (vid. Loesch, 1965). Habrá que buscar en otros textos y/o épocas para hallar títulos absolutamente idioculturales ${ }^{28}$.

El problema radica, más bien, en las fórmulas de tratamiento, que pueden invitar a la apropiación traductológica o, como defendemos, servirse de la substitución cultural para evitar hiatos comunicativos con el público presente en el teatro. Veamos algunos ejemplos.

\subsection{Análisis y propuesta de translación de las fórmulas de tratamiento}

Pasemos a analizar las fórmulas de tratamiento y sus posibles traducciones, en bloques: según destinatario y según calidad.

3.3.1. Fórmulas de tratamiento entre iguales y de inferior a superior

\section{A new way to pay old debts}

Sir

Se propone «Señor» como la única fórmula de tratamiento, tanto entre pares como de inferior a superior.

\section{Your Grace}

Es fórmula de tratamiento de obispos y de duques, incluidos los hermanos del monarca, como decíamos en la Introducción. Se propone «Vuecencia», al no ser de familia real y a pesar del carácter irónico de la cita.

\section{Your worship/worshipful/right worshipful}

\footnotetext{
${ }^{28}$ Para un listado comprehensivo de títulos, tratamientos y demás elementos de etiqueta en la cultura británica de hoy en día se puede consultar el Debretts, también disponible en línea.
} 
A finales del siglo XVII, la fórmula de tratamiento your worship y sus derivados worshipful y right worshipful eran de apelación genérica; por lo que pueden substituirse por «Vuesamerced». Posteriormente, se restringiría a jueces, jueces de paz, concejales y otras dignidades judiciales y administrativas civiles que, en español, normalmente, tendrían tratamiento, como mucho, de "Señoría". Comoquiera que coincide en el tiempo y se solapa con las fórmulas de tratamiento de la nobleza, se ha tomado la decisión de substituir your worship y worshipful por «Señoría» y right worshipful por «llustrísimo Señor» o «llustrísima».

\section{Right honourable}

Lord Lovell pertenece a la baja nobleza: es vizconde o barón y, como tal, tiene derecho al tratamiento de Right honourable que, en español, equivaldría a «llustrísimo Señor» en el caso de los vizcondes y a «Señor» en el de los barones ${ }^{29}$.

\section{Fellow ${ }^{30}$}

AMBLE.-Why, fellow Furnace, 'tis not twelve o'clock yet, Nor dinner taking up; then, 'tis allow'd, (I, ii, 10-11)

Se traslada por «camarada», que es tratamiento coetáneo entre pares.

\section{Goodman (y, en su caso, Goodwife o Goody)}

FURNACE.-You think you have spoken wisely, goodman Amble, My lady's go-before! (I, ii, 13-14)

El compuesto aparece una sola vez en toda la obra. Es fórmula de tratamiento entre plebeyos y burgueses y se asocia con las clases medias emergentes, con el puritanismo inglés y/o presbiterianismo escocés. El ejemplo literario más conocido desde mediados del siglo XX, es The crucible («Las brujas de Salem») de Arthur Miller, que transcurre en la colonia puritana de la Bahía de Massachusetts, entre 1692 y 1693. No hay realmente una equivalencia aceptada en español para Goodman y Goody. En la traducción que llevaran a cabo de The Crucible Jacobo y Mario Muchnik, en 1955, por ejemplo, Goody, seguido de apellido, se reemplaza por "la señora X", si es que llega a reflejarse. Aquí como vemos, Amble, el ujier, es apelado como Goodman Amble. Lo habitual habría sido recurrir al Vuesamerced/Su

\footnotetext{
${ }^{29}$ Ver el párrafo anterior sobre el solapamiento con los títulos y fórmulas de tratamiento en los dignatarios del legislativo y judicial y las decisiones tomadas al respecto.

${ }^{30} \mathrm{Al}$ igual que haremos más adelante con los apelativos y fórmulas burlescos, damos la cita por la polisemia de estos lemas.
} 
Merced/Usarced ${ }^{\beta 1}$ y demás variantes (incluido voacé y otros), de aceptación indistinta en los diversos estamentos, incluso como apelativo entre los criados y hasta entre delincuentes, como apuntaba Stevens en 1706 y recordará Lapesa en 194232. Pecando de filólogos, podría tentarnos substituir Goodman por «Guzmán», ya que el apellido español proviene etimológicamente de las mismas raíces germánicas - gut «bueno» y mann «ser humano, hombre»como bien observara Covarrubias en su Tesoro y nos ratificaron Faure, Ribes y García (2006, pg. 405) casi cuatrocientos años después. Pero sería una pirueta en el vacío y sin red: además lo vale para el hombre no valdría, en su caso, para la mujer y, sobre ese inconveniente, Guzmán nunca ha sido fórmula de tratamiento. Tampoco nos valdría el vocativo buen hombre y/o buena mujer, históricamente tan común para dirigirse a las clases populares cuando el apelador desconoce el nombre y/o apellidos del apelado. Solamente nos queda un resquicio que cumple con el condicionante primero, el del tiempo y, además, se ajusta a las premisas de traducción literaria propuestas en su día por Jiři Levý (1969: 68-71) y por Katharina Reiss (1971, pp. 37-44, 1976, pp 13 s.). Se trataría de proponer seor (que no sor) para Goodman y, en su caso, seora para Goody (< Goodwife). Hay coincidencia de sociolecto con los originales en cuanto a tratamiento de clases burguesas y/o entre menestrales en la Edad Moderna: desde mediados del siglo XVI hasta el siglo XVIII, concretamente de 1577 a 1732 para Goodman y 1559 a 1798 para Goody (OED). También coinciden con el nivel de formalismo

31 Esta última la seguirá registrando CNDHE hasta finales del siglo XIX en texto peruanos, vgr. 1889, Ricardo Palma, Tradiciones peruanas, séptima serie [Madrid, EspasaCalpe, 1969]. Según el CNDHE, la apocopada usarcé saltará desde Zorrilla El zapatero y el rey en 1840 hasta dos citas cubanas de 1963, de la mano de Fernando Ortiz Contrapunteo cubano del tabaco y el azúcar [Julio Le Riverend, Caracas (Venezuela), Ayacucho, 1987]. La otra variante, voacé, conocida, sobre todo, por Cervantes y su Al Túmulo del rey que se hizo en Sevilla, la registran CNDHE y CORDE desde los Romances de Góngora (1580-1627) hasta 1673 en la comedia El hidalgo de la Mancha: jornada primera de Juan de Matos Fragoso.

32 Stevens (1706), A new Spanish and English Dictionary. Collected from the best Spanish Authors both Ancient and Modern (...) To which is added a Copious English and Spanish Dictionary. London. George Sawbridge:

«Voacé or Voarcé, us'd by Ruffians and Bullies, and sometimes by Country Fellows for Uste, or Vuéstra Mercéd"

y Rafael Lapesa Melgar 1942, Historia de la lengua española [España] [ Madrid, Escelicer, 1942]

«La etiqueta y puntillosidad de nuestros antepasados creyó insuficiente el tú de la intimidad familiar o el vos, tan corriente que era descortesía emplearlo con quien no fuese allegado o inferior. De no ser así, había que tratar de vuestra merced o vuestra señoría, y la repetición originó el paso de vuestra merced a vuessa merced, vuesarced, vuesançed, etc., y finalmente a voacé, vucé, vuced, vusted, usted; en el siglo XVII estas últimas formas eran propias de criados y bravucones, pero, a pesar de ello, acabaron por generalizarse. De igual manera (...) señor, colocado como título delante de un nombre o adjetivo, degeneró en seor, seó y so (so gandul, so pícaro, del lenguaje vulgar).»

Hikma 18 (2) (2019), 37 - 65 
lingüístico, con la diastratía. Incluso 'concuerdan fonéticamente' en tanto en cuanto goody es síncopa de goodwife y seora ${ }^{33}$ lo es de señora. Desde el punto del condicionante temporal, de la apetecida isocronía, no había problema alguno con seor. Antepuesto a apellido tanto CNDHE como CORDE dan ca. 1600 y el anónimo, Entremés de la cárcel de Sevilla: «PAISANO. Pues dé la sentencia el seor Barragán, que es hombre que a todos los hombres del mundo les puede meter la baraja en la boca.» Como vocativo, CNDHE lo da ibídem: «PAISANO. Sí, seor.» Antepuesto a título hereditario CNDHE lo da ca. 1623, Luis Quiñones de Benavente El marqués de Fuenlabrada: «PIC[ADOR] ¿Está acá el seor Marqués de Fuenlabrada?» Antepuesto a los agnominales respiciales de todo tipo (militar, curial, académico, profesional, parental, etc.), las citas se multiplican passim. Tampoco habría problemas con seora, si bien CNDHE no lo ofrece como lema autónomo y NTLLE no lo registra antes del Diccionario Usual de 1884. CORDE nos lo da ca. 1625, antepuesto a nombre de pila, como «seora Toribia» en Luis Quiñones de Benavente, Otañez y el Fariseo; como vocativo en 1656, «Ah seora, pase adelante» en Francisco Bernáldez de Quirón, Aventuras de Don Fruela; y, antepuesto a fórmula de tratamiento y apellidos, ca. 1658, en el Entremés del comendador de Pedro Calderón de la Barca. La última cita en CORDE es de 1763 y Ramón de la Cruz en La víspera de San Pedro y la de CNDHE es de ca. 1765 y el anónimo La maja. Tonadilla a solo: «Seora Marianita.»

The country wife

Lady

Como título cognominal o agnominal, Lady puede ser apropiado o substituido por Doña, como ya se ha dicho arriba. Por el contrario, en tanto que fórmula de tratamiento, proponemos el galicismo «Madama», que registra 'Autoridades' y se documenta por vez primera en la Edad Media tardía (1377-1393) ${ }^{34}$, siendo común hasta el siglo XIX.

\section{Master}

Al haber aceptado verter Mr. como «Maese», para master no nos quedaba sino "señorito". En los corpora españoles la primera cita es anónima, de 1526, fragmento (Marcos de Aguilar, Carta del licenciado Marcos de

\footnotetext{
${ }^{33}$ Incluso sora —en 1604 y La pícara Justina, por ejemplo- aunque CNDHE apenas registre la síncopa y haya que recurrir a CORDE.

${ }^{34}$ En el CNDHE, las 58 primeras citas del lema lo son de la obra de Juan Fernández de Heredia, Crónica de Morea [Juan Manuel Cacho Blecua, Zaragoza, Universidad de Zaragoza, 2003] y arrancan con: «Et muerto el dicho Roberto, los uarones del imperio enuiaron a França et fizieron uenir una su hermana del dicho emperador Baldoyn et su ermano Ruberto, la qual se clamaua madama Hyola, la qual era muller de miçer Pero Dagon, conde de Aniou.»
} 
Aguilar, y documentos anexos): "Capítulo 9. Que al repartir de la tierra se tenga mucho respeto a los niños y señoritos que son en los monasterios". Como fórmula de tratamiento es de c1560 - a1578, en las Poesías de Francisco de Aldana: «sálveos Dios, mis señoritos» en plural y de c1565, en la Comedia de Sepúlveda de Lorenzo Sepúlveda: «Hazeos allá, que no me beáis... ¡Más allá, señorito, que tantas ruindades sé como vos!» en singular. La primera cita que ofrece OED es coeva (c1533-4, H. Dowes Let. in H. Ellis Orig. Lett. Eng. Hist. (1846) 3rd Ser. I. 343: «It pleased your Maistershipp to give me in charge not onlie to give diligent attendaunce uppon Maister Gregory»), si bien, en inglés y hasta 1720, suele aparecer en el sintagma nominal young Master, como por ejemplo,1600, Shakespeare, Merchant of Venice II. ii. 44: «Talke you of young Maister Launcelet».

\section{Mistress}

El sentido válido en nuestro corpus para Mistress es «dueña/Doña» (< latín domina), lema que es equiparable a la evolución inglesa del francés antiguo maistresse (< latín magistra), que se certifica en el OED como «esposa del amo/dueño» a1375 (William of Palerne (1867) 1016 (MED): «Alisaundrine..attlede pe sope, pat hire maistres \& pat man no schuld hire nou3t misse, pezh sche walked..from here si3t») y como "ama/dueña" a1393 (Gower, Confessio Amantis (Fairf.) I. 1825 (MED): «Sey what you list in my querele..evere whil that I may live, I wol that ye be my maistresse»). Como fórmula de tratamiento, se origina en Chaucer, ?a1425 ( c1380) y su traducción de Boecio, De Consolatione Philos. I. pr. iv. 161: «O Maystresse, what demestow of this?».

\section{Madam}

Madam vale «Señora», sin problemas.

Your Ladyship

Your Ladyship vale "Vueseñoría", sin problemas.

\section{Love for Love}

\section{Miss}

El lema $M /$ miss, apocope de Mistress, lo data OED por vez primera en 1606, significando "concubina, barragana»: Nicholas Breton, Choice, Chance, \& Change or, Conceites in their colours, sig. H2: «If your mistris haue a fine wit, and your wife, but a plaine vnderstanding (...) if your mis. Be kind \& your wife dogged: will you loue your mis. Better than your wife?». Como título agnominal antepuesto a apellido, lo data en la entrada del Diary de Samuel Pepys de 7 de marzo de 1667 ((ed. 1974) VIII, 101: «Little Miss 
Davis»). Antepuesto a nombre de pila, no lo registrará hasta 1745 (Jonathan Swift, Direct. To Servants, 93: «Miss Betty won't take to her Book»). Cómo fórmula de tratamiento, que es la que nos interesa aquí, también se data de 1667 en Dryden (The Maiden Queen III: «Adieu, dear Miss! If ever I am false to thee again»). Como nombre, en español ya aparece en 1495 y Nebrija: «Damicela casi señorita. Domicella, no». Como título agnominal ante nombre de pila, en 1806, Leandro Fernández de Moratín todavía se servirá del tradicional Doña para el personaje de Doña Francisca/Paquita de El sí de las niñas. La Academia no registra señorita hasta el Diccionario Usual de 1899.

No es fácil, por tanto, la substitución cultural. Si prescindimos de un Doña antepuesto al nombre de pila, podríamos proponer "doncella" o "niña". En boca de Tattle podría valer "doncella" o cualquiera de sus diminutivos. En boca de Mrs. Frail o de la Nodriza, entendemos que la mejor opción sería "Niña", que, por lo demás, figura en oración directa, como vocativo, desde c1275 [principios del s. XIV], en la 'Segunda Parte' de la General Estoria de Alfonso X 'El Sabio': "O tu quien quier que eres, sal aca. Et tu ninna aun sola por que me engañas» y es muy común en toda la Edad Moderna.

\subsubsection{Tratamientos vejatorios e insultantes}

El segundo bloque se contrapone al primero al listar tres fórmulas ambiguas de tratamiento que pueden ser afrentosas, neutras o incluso afectuosas, ambas extraídas de Love for Love y tres afrentosas de The Country Wife y de Love for Love.

\section{Rascal}

Rascal vale «bribón» (así lo vierte Astrana en MM v, i, 301) y cumple con la coherencia temporal, al estar documentado como apóstrofe - «bribón troyano»— desde 1597-1645, en la obra poética de Francisco de Quevedo.

\section{Rogue}

Se propone «pícaro», aunque valdría «bribón» ... pero no «tunante», que sólo a finales del siglo XVIII (1796, Anónimo, La maja alegre. Tonadilla a tres: «ELLA. ¡Tunante!») lo citará CNDHE en oración directa. De naturaleza despreciativa, pero también afectuosa ${ }^{35}$ en boca de superior a inferior, el nombre rogue surge en la germanía con el sentido de «vagabundo» (1489, Caxton tr. C. de Pisan, Book Fayttes of Armes ii. ix. sig. Gvi: «He came bifore roges whiche he knewe wel garnisshed of vitaille, for the whiche thing they kept hem stronge») al que se agregará el sentido genérico de «pícaro» (1568, W. T. Ritchie, Bannatyne MS f. 210v/5: «Som rakles roig may hasard hir to

${ }^{35}$ Según Onions, rogue en $2^{\text {a }}$ acepción, vale rascal «applied abusively to servants», pero también será «term of endearment», en $3^{3}$ acepción y con mayor número de citas. 
ryde And namlie at ane anker in the night.»). A la hora del traslado, la duda estaba entre dos términos coevales: «pícaro», que también permite el afectivo picar(ón/ona) o picaruel(o/a) y «bribón», con su correspondientes brib(ón/ona) y briboncill(o/a)/bribonzuel(o/a). La ventaja del primero estriba en que, para los diccionarios de los siglos XVI y XVII, pícaro vale «vagabundo», como el primer significado monolingüe de rogue; además, en el diccionario de Nicholas Mez von Braidenbach (1670), pícaro vale Halunck o Spitzbub (NTLLE) y rogue es Haluncke en alemán. En la terminología literaria, el español pícaro se suele traducir por rogue y Mabbe propuso como traducción "The Rogue» para La vida del pícaro Guzmán de Alfarache de Mateo Alemán en 1622.

\section{Sirrah}

Se propone «bellaco», que, al igual que el lema inglés ${ }^{36}$, podía ser tanto ofensivo como familiar y afectuoso en el trato de superiores a inferiores; e incluso, como nombre común hasta cierto punto positivo, según 'Autoridades'37.

\section{The country wife}

\section{Wench}

Se propone «barragana» 0, preferiblemente, «manceba», por coherencia temporal y por evolución comparable en ambos casos. Desde el inglés antiguo wencel hasta mediados del siglo XIV, el lema valía «hembra», para pasar, ya con apócope, a "moza, criada" o "joven" a partir de 1290. En 1362, (Piers Plowman A 'Prologue' 51: "Ermytes on an hep wit hoteide staues Wenten to Walsyngham \& here wenchis aftir»), se empezará a usar en referencia a rameras o a concubinas. Es una evolución de envilecimiento similar y paralela en el tiempo a la del francés fille (< latín filia), que hará necesario, en su momento, especificar jeune fille ${ }^{38}$.

\footnotetext{
${ }^{36}$ Según Onions, sirrah es "ordinary form of address to inferiors".

${ }^{37}$ Astrana vierte sirrah de manera muy diversa: "compañero" (AYL IV, iii, 167), "pícaro" (AYL III, ii, 169; $1 \mathrm{H} 4 \mathrm{I}$, ii, 200), "pillo" (2H4 V, iii, 16) y, por dos veces, "compadre" en boca de Capuleto (RJ I, v, 33 y 130).

38 cf. 1389 "femme qui mène une vie de débauche " (Ordonnances des rois de France de la troisième race, t. 7, pp. 309: fille de joye). Recuperado de http://atilf.atilf.fr/dendien/scripts/ltfiv5/affart.exe?19;s=1871716575;?b=0).

Por lo demás, sí que es cierto que, desde finales del siglo XVI hasta mediados del XIX, el OED registra wench con un valor afectuoso y familiar equiparable al de rogue, también en Shakespeare, en su teatro como en las obras poéticas (Tp. I, ii, 139; H8 III, i, i; Lucr. 1273). Para el OED, la primera datación con tal sentido es: 1581, A. Hall, y su traducción de Homero 10 Bks. lliades v. 97 [Juno to Pallas]: «Go we my wench, and let vs shew this dizarde here at ful, What power. we two haue».
} 


\section{Love for love}

\section{Hussy}

La síncopa hussy deriva del inglés antiguo huswif «housewife» con envilecimiento desde 1647. En nuestra obra se puede usar como insulto, pero a la vez, como un apelativo familiar y casi cariñoso, valor que el OED registra desde 1650. Nuestra propuesta, «mujercilla», puede tener ese sentido ofensivo en las referencias coetáneas ${ }^{39}$, pero es preferible al mucho más radical "mujerzuela", que sólo está presente una vez antes del siglo XVIII, c1632, en La Perinola de Francisco de Quevedo: "¿Qué terremotos de imaginaciones formará en los entendimientos de un oficialejo y de una mujerzuela este Dios multiplicado, y este una vez Dios y dos veces Dios?».

\section{Slut}

Slut es «puta rastrera y sucia». Con «sota» proponemos una imitación fonética de válida coherencia temporal, pues ya aparece en CNDHE con ese valor de 1585 - a1643, en la obra poética de Juan de Salinas — «algunos la llaman sota»- y en la cita quevediana que aporta Hernández Castanedo (1994: 240 s.).

\subsubsection{Tratamientos burlescos y paródicos}

Finalizamos con los tratamientos espurios y lúdicos. Aquí orillamos nuestras lindes autoimpuestas de erigir equivalentes coetáneos en la cultura española. No tiene sentido rastrear una contraposición históricamente coherente de lo que no pasa de ser una chanza neologizante, de lemas que no indican sino evocan, que no muestran, que disfrazan. Ahora bien, por la misma razón, como establece Newmark (1988, pg. 143):

In principle, in fiction, any kind of neologism should be recreated; if it is a derived word it should be replaced by the same or equivalent morphemes; if it is also phonoaesthetic, it should be given phonemes reproducing analogous sound effects. (...) the neologisms (...) must be recreated systematically and ingeniously, always however with the principle of equivalent naturalness in mind.

\footnotetext{
39 Tiene valor peyorativo desde la primera datación a1260 [mediados del s. XIII], Anónimo, El Nuevo Testamento según el manuscrito escurialense 1-j-6. Desde el Evangelio de San Marcos hasta el Apocalipsis [España] [Thomas Montgomery/Spurgeon W. Baldwin, Madrid, Real Academia Española, 1970]: «mugerciellas cargadas de peccados»; pero, en la Edad Moderna, puede valer simplemente como diminutivo. Como fórmula de tratamiento, cf. 1597, Francisco Narváez de Velilla, Diálogo intitulado el capón: «¿Qué quieres, mujercilla?»
} 
El reto se torna puramente poético, es decir creativo: inventar un neologismo capaz, como en los demás casos, de provocar una reacción similar en el espectador.

A new way to pay old debts

Your Mastership

TAPWELL.- True, but they brought in profit,

And had a gift to pay for what they call'd for,

And stuck not like your mastership. (I, i, 75-79)40

A lo largo de la obra abundan los tratamientos puramente jocosos y/o imitativos de los existentes en la sociedad barroca inglesa. Aquí se propone «Vuesa Maestría».

Your Solicitorship

OVERREACH.- And yet your good solicitorship, and rogue Wellborn,

Were brought into her presence, feasted with her! (II, iii, 115-116)

Es imitación burlona de lordship «señoría». OED da esta cita como hápax, por lo que hemos recurrido a un neologismo imitativo: a partir de procurador ... "Vuesa Procurancía», puesto que la recreación morfológicamente más cercana, procuraduría, ya existe con sentido establecido.

His wise head

WELLBORN.- I heard of him, madam,

By his minister, Marrall; he's grown into strange passions

About his daughter. This last night he look'd for

Your lordship at his house, but missing you 96

And she not yet appearing, his wise head

Is much perplex'd and troubl'd. (V, i, 93-98)

El compuesto neológico wise head que se asocia etimológicamente a un *wisehood, como forma alternativa de wisdom «sabiduría, prudencia» (< inglés antiguo wīsdōm), ${ }^{41}$ es una clara alusión satírica que el OED primo-

${ }^{40}$ Dado el carácter extraordinario, incluso el valor de hápax, de estos translemas, se insertan las citas para mejor explicitación co-textual.

${ }^{41}$ Compárese el origen común de maidenhead (que derivará metonímicamente en «himen») y maidenhood «virginidad», ambas derivadas del inglés antiguo mæzdenhād «doncellez» y donde 
datará más de un siglo después (1756, Monitor 64 II 122) como sintagma nominal y no como fórmula de tratamiento, saltándose la obra que nos ocupa. Puestos a recrear morfológicamente un neologismo equivalente hay que oponer el sufijo abstracto de cualidad español -dad (o -edad, -idad, -bilidad) y, al mismo tiempo, dar la sensación de naturalidad lingüística, como pedía Newmark arriba. Dado que, posiblemente, el sinónimo coetáneo de 'sabiduría' más común fuera 'prudencia' que, sobre ser virtud cardinal, como nos recuerda Covarrubias, aparece en obras como La prudencia en la mujer de Tirso de Molina (1622) y otras similares, se propone el neologizante y burlesco «Su Prudencidad».

\section{The country wife}

\section{Reverend ladies}

HORNER.- I thank you Madam, I could never find pitty, but from such reverend Ladies as you are, the young ones will never spare a man. (IV, iii)

Se propone "Damas de vuestra reverencia», en vez de "reverendas damas» y, mucho menos, «reverendas». Se intenta conservar la ironía y se le añade una asociación con la fórmula de tratamiento "Vuestra Reverencia» que, en la cultura española coetánea, se reservaba para personas profesas en religión y, por lo tanto, célibes: «A todos estos por cortesía ponemos (...) 'Padre Maestro' al religioso, 'Vuestra Paternidad' al más grave, i perlados, 'Vuestra Rreverenzia' á los demas», como decía Correas (1954, pg. 213).

\section{CONCLUSIONES}

De las treinta unidades, la mayoría absoluta han podido ser resueltas de acuerdo con nuestras premisas, puesto que solamente se han apropiado tres títulos: Lord, Lady y Sir. En lo que respecta a los demás, se ha procedido a la triangulación/traducción semántica, más o menos libre, de once de ellos, incluidos los tratamientos afrentosos y los neologismos para responder a los tratamientos burlescos: Sir/Señor, Fellow/Camarada, Rascal/Bribón, Rogue/Pícaro, Sirrah/Bellaco, Wench/Manceba, Hussy/Mujercilla, Your Mastership/Vuesa Maestría, Your Solicitorship/Vuesa Procurancía, His Wise head/Su Prudencidad, Reverend ladies/Damas de vuestra reverencia. A nuestro juicio, la substitución ha valido, con mayor o menor fortuna -

el sufijo de hād derivará, por evolución fonética regular, en el sufijo abstracto de cualidad -hood que, en el inglés de la Edad Moderna Temprana, se prestaba a confusión. Así se demuestra en el juego de palabras entre los dos criados de la casa de Capuleto: «SAMPSON: I will be cruel with the maids; I will cut off their heads. GREGORY: The heads of the maids? SAMPSON: Ay, the heads of the maids, or their maidenheads» (RJ I, i, 26-30), obra compuesta entre $1594 \mathrm{y}$ 1596. 
forzando la mano un tanto en casos puntuales como Miss y Niña - para los dieciseis restantes, en más del 50\% de los ejemplos: Gentleman/Hidalgo, Mr./Maese, Mrs./Doña, Your Grace/Vuecencia, Lady/Madama, Your Ladyship/Señoría, Your Worship/Señoría, Worshipful/Señoría, Right Worshipful/llustrísima, Right Honourable/llustrísimo Señor, Goodman o Goodwife/Vuesa Merced, Master/Señorito, Mistress/Dueña o Doña, Madam/Señora, Miss/Niña y Slut/Sota.

Por todo ello, nos mostramos confiados en la validez de nuestra propuesta metodológica para la traducción de la dramaturgia no contemporánea en el contraste de la cultura inglesa y la española de la Edad Moderna.

\section{REFERENCIAS BIBLIOGRÁFICAS}

Referencias primarias (obras literarias originales y traducciones)

Congreve, William. (1968). Love for love. (Edited with an introduction by Gāmini Salgādo). Harmondsworth: Penguin.

Massinger, Philip. (2000). A New Way to pay old Debts. En English Renaissance Drama. A Norton Anthology. (David Bevington general editor). New York: W.W. Norton.

- (2001). A New Way to Pay Old Debts. Vol. XLVII, Part 5. The Harvard Classics. New York: P.F. Collier \& Son, 1909-14; Bartleby.com. 2001. Recuperado de: www.bartleby.com/47/5/.

Miller, Arthur. (1959) [1953]. The crucible. A play in four acts. New York: Bantam Books. [La primera traducción española es la de Jacobo y Mario Muchnik, Las brujas de Salem. Buenos Aires: Muchnik editor. 1955]

Shakespeare, William. (1974). Complete Works. (W.J. Craig ed.). Oxford: Oxford University Press.

- Astrana Marín, Luis. $1974^{16}$ [1932]. William Shakespeare. Obras completas. (Estudio preliminar, traducción y notas de Luis Astrana Marín. Primera versión íntegra del inglés). 2 tomos. Madrid: Aguilar.

— Rusconi, Carlo. (1858). Teatro completo de Shakespeare. Recuperado de: bit.ly/2WTZEhz

— Schlegel, Friedrich, Wolf Graf Baudissin et al. (s/d). William Shakespeare. Gesamte Werke. Wiesbaden: R. Löwit GmbH. 
- Victor-Hugo, François. (1961, tomos I y II; 1964, tomo III) Shakespeare. Théàtre complet. (Introduction, notices et notes par J.B. Fort, Professeur à la Sorbonne). 3 tomes. Paris: Garnier Frères.

Wycherley, William. (1968). The country wife. (Edited with an introduction by Gāmini Salgādo). Harmondsworth: Penguin.

- The country wife. Edición en línea en: Early English Texts: https://quod.lib.umich.edu/e/eebo/A67192.0001.001/1:4.1?rgn=div2;vi ew=fulltext. (Consultado por última vez 23/10/2018).

Referencias secundarias

Brown, Roger y Albert Gilman. (1960). «The pronouns of solidarity and power», en Thomas Albert Sebeok. (Ed.). Style in Language, reeditado en Pier Paolo Giglioli, (Ed.). (1972). Language and social context, Harmondsworth: Penguin (Pelican), pgs. 252-282.

Calvo García de Leonardo, Juan José. (2013). «BSOs, subtitulados, trampantojos y saborizantes: la eponimia en los textos de turismo", en El lenguaje del turismo y la publicidad: terminología y traducción. ePraxis (plataforma del grupo editorial Wolter Kluwer España, S.A.), pgs. 31-57.

Catford, John Cunnison (lan). (1965). A linguistic theory of translation. Oxford: Oxford University Press.

Cicero, Marcus Tullius. (1964). «De optimo genere oratorum», en Rhetorica II (A.S. Wilkins, ed.). Oxford. At the Clarendon Press (script. class. bibliotheca oxoniensis).

Correas, Gonzalo. (1954). Arte de la lengua española castellana. (Edición y prólogo de Emilio Alarcos García). Madrid: CSIC (RFE: Anejo 56).

Dagut, Menachem. (1978). Hebrew-English Translation: A linguistic analysis of some semantic problems. Haifa: University of Haifa.

Fernández Fernández, Francisco, Miguel Fuster Márquez y Juan José Calvo García De Leonardo. (2001). Early Modern English Texts. Valencia: Albatros.

Gerhard, Sandra Forbes. (1982). Don Quixote and the Shelton translation. A stylistic analysis. Potomac (Md): Studia Humanitatis.

Holmes, James Stratton. (1978), «Describing literary translations: models and methods», en James Stratton Holmes, José Lambert, y Raymond Van Den Broeck. (Eds). (1978). Literature and translation. New perspectives in literary studies. Leuven: ACCO, pgs. 69-82. 
Koller, Werner. (1979). Einführung in die Übersetzungswissenschaft. Heidelberg: Quelle und Meyer.

Koschmieder, Erwin. (1965). Beiträge zur allgemeinen Syntax. Heidelberg: Winter.

Levý, Jiři. (1969). Die literarische Übersetzung. Theorie einer Kunstgattung. (Traducción alemana de Walter Schamsula del original checo de 1963). Frankfurt am Main: Athenäum. [Hay traducción inglesa: The art of translation. Translated by Patrick Corness. Edited with a critical foreword by Zuzana Jettmarová. Amsterdam: John Benjamins. 2011].

Loesch, Ilse. (1965). So war es Sitte in der Renaissance. Hanau M: Verlag Werner Dausien.

Neubert, Albrecht. (1968). «Pragmatische Aspekte der Übersetzung», en Albrecht Neubert. (Ed). Grundfragen der Übersetzungswissenschaft. Leipzig: Beihefte zur Zeitschrift Fremdsprachen, pgs.21-33.

Newmark, Peter. (1988). A textbook of translation. London/New York: Prentice Hall.

Nida, Eugene Albert. (2012). Sobre la traducción. (Selección y traducción el autor y de M. Elena Fernández Miranda-Nida). Madrid: Cátedra.

Nida, Eugene Albert y Charles Russell Taber. (1969). The theory and practice of translating. Leiden: Brill.

Ogden, Charles Kay e Ivor Armstrong Richards (1923). The Meaning of Meaning: A Study of the Influence of Language upon Thought and of the Science of Symbolism. London: Kegan, Paul, Trench, Trubner.

Reiss, Katharina. (1971). Möglichkeiten und Grenzen der Übersetzungskritik. Kategorien und Kriterien für eine sachgerechte Beurteilung von Übersetzungen. München: Max Hueber.

- (1976). Texttyp und Übersetzungsmethode. Der operative Text. Kronenberg TS: Scriptor.

Reiss, Katharina y Hans Josef Vermeer. (1996). Fundamentos para una teoría funcional de la traducción. Madrid: Akal (Universitaria).

Santoyo, Julio César. (1986). «A propósito del término TRANSLEMA», en Actas del I congreso nacional de AESLA. Murcia, 14-17 de abril de 1983. Madrid: AESLA-Sgel: pgs. 255-265. 
Schleiermacher, Friedrich. (1969). «Das Problem des Übersetzens», en Hans Joachim Störig. (Ed.). Das Problem des Übersetzens. Stuttgart/Darmstadt: Wissenschaftliche Buchgesellschaft, pgs. 38-70.

Ullmann, Stephen $\left(1957^{2}\right)$ [1951]. The principles of semantics. Glasgow University publications LXXXIV. Oxford: Basil Blackwell.

Vega, Miguel Ángel. (Ed.). (1994). Textos clásicos de teoría de traducción. Madrid: Cátedra.

Vinay, Jean Paul y Paul Darbelnet (1958). Stylistique comparée du français et de l'anglais. Methode de traduction, Paris: Didier.

Wilss, Wolfram. (1977). Übersetzungswissenschaft. Probleme und Methoden. Stuttgart: Klett.

Diccionarios y glosarios

Cobarruvias Orozco, Sebastián de. (1977). Tesoro de la lengua castellana o española. Madrid: Ediciones Turner.

Collins Cobuild English Dictionary. (1987). London \& Glasgow: Collins.

Corominas, Joan. (1955-1957). (3ª reimpresión, marzo 1976). Diccionario crítico-etimológico de la lengua castellana. 4 vols. Madrid: Gredos.

Drosdowski, Günther y Paul Grebe. (1963). Der Grosse Duden 7. Herkunftswörterbuch. Die Etymologie der deutschen Sprache. Mannheim, Wien y Zürich: Bibliographisches Institut AG Dudenverlag.

Faure Sabater, Roberto, Maㅡ Asunción Ribes y Antonio García. (20064). Diccionario de apellidos españoles. Madrid: Editorial Espasa-Calpe.

Hernández Castanedo, Francisco. (1994). Glosario de la mala palabra (De los mil y pico nombres con que atienden las del más viejo oficio). Madrid: Editorial El Avapiés.

Lorenzo, Emilio. (1996). Anglicismos hispánicos. Madrid: Gredos.

Nebrija, Antonio de. (1981). Vocabulario de romance en latín. (Transcripción crítica de la edición revisada por autor (Sevilla, 1516) con una introducción de Gerald J. MacDonald). Madrid: Editorial Castalia.

Onions, Charles Talbut. (1978) [1919²]. A Shakespeare Glossary. Oxford: At the Clarendon Press.

Onions, Charles Talbut, George Washington Salisbury Friedrichsen y Robert William Burchfield. (1966). The Oxford dictionary of English etymology. (1969, 3ª reimpresión corregida). Oxford: Oxford University Press. 
Oxford English Dictionary. (1971). The compact edition of the complete text reproduced micrographically. 2 vol. (1984, 23 ${ }^{\text {rd }}$ printing). Oxford: University Press. Complete text reproduced micrographically. Vol. 3 (1987). A supplement to The Oxford English Dictionary Volumes I-IV. Oxford: At the Clarendon Press.

Real Academia Española. 1963. (3ª reimpresión, febrero 1976). Diccionario de Autoridades (edición facsímil). 3 vols. Madrid: Gredos.

Real Academia Española. (201423). Diccionario de la lengua española. (Edición del tricentenario. 1aㅡ tirada: octubre de 2014). Madrid: Espasa Libros.

Rodríguez González, Félix (Dir.) y Antonio Lillo Buades. (1997). Nuevo diccionario de anglicismos. Madrid: Gredos.

Shuttleworth, Mark y Moira Cowie. (2014). Dictionary of translation studies. Abingdon \& New York: Routledge.

Skeat, Walter William. (1879-1882). An etymological dictionary of the English Language. (reimpresión 1988) Oxford: At the Clarendon Press.

Referencias en línea

DEBRETTS. Recuperado de: https://www.debretts.com/expertise/forms-ofaddress/

Digitales Wörterbuch der deutschen Sprache (DWS). Recuperado de: https://www.dwds.de/.

Campillo Arnaiz, Laura. (2005). Estudio de los elementos culturales en las obras de Shakespeare y sus traducciones al español por Macpherson, Astrana y Valverde. Tesis doctoral recuperada de: https://tesisenred.net/handle/10803/10813.

Grimm, Jacob y Wilhelm Grimm. Deutsches Wörterbuch. Recuperado de http://woerterbuchnetz.de/DWB/.

Littré, Emile. (1872-1877). Dictionnaire de la langue française, en Dictionnaires d'autrefois. Recuperado de: http://artflproject.uchicago.edu/node/17.

Mazzon, Gabriella. (2010). "Address terms", en Andreas H. Jucker e Irma Taatvitsainen (eds.). Historical pragmatics. (Handbook of Pragmatics. 8. Editores Wolfram Bublitz, Andreas H. Jucker y Klaus P. Schneider) Berlin: De Gruyter/Mouton, pgs. 351-378. Recuperado de: http://bit.ly/34lqEDe. 
Medina Morales, Francisca. Las formas nominales de tratamiento en el Siglo de Oro. Aproximación sociolingüística. Recuperado de: https://cvc.cervantes.es/literatura/aiso/pdf/06/aiso_6_2_033.pdf.

Nelson, Walter. Forms of address in Tudor England. Recuperado de: walternelson.com/dr/node/253.

Oinonen, Raisa. (2012). "Yours to command": Politeness and an Early Modern English subscription formula. Recuperado de: www.helsinki.fi/varieng/series/volumes/11/oinonen/.

Real Academia Española. Nuevo tesoro lexicográfico de la lengua española (NTLLE). Recuperado de: http://buscon.rae.es/ntlle/SrvltGUILoginNtlle.

Real Academia Española. Corpus del nuevo diccionario histórico del español (CNDHE). Recuperado de: web.frl.es/CNDHE/view/inicioExterno.view.

Real Academia Española. Corpus diacrónico del español (CORDE). Recuperado de: http://corpus.rae.es/cordenet.html.

Secara, Maggie. (2011)11 (Spring 2011, newly expanded) "Titles and forms of address" en Life in Elizabethan England. A compendium of common knowledge.

Recuperado de: www.elizabethan.org/compendium/home.html.

Taavitsainen, Irma y Andreas H. Jucker. (Eds.). (2003). Diachronic perspectives on address terms systems. Amsterdam/Philadelphia: John Benjamins. Recuperado de: http://bit.ly/2WUOVVT. 